\title{
NONLOCAL INTERACTIONS AND QUANTUM DYNAMICS
}

\author{
Renat Kh.Gainutdinov \\ Department of Physics \\ Kazan State University, \\ 18 Kremlevskaya St, Kazan 420008, \\ Russia \\ E-mail: Renat.Gainutdinov@ksu.ru
}

\begin{abstract}
The problem is considered of describing the dynamics of quantum systems generated by a nonlocal in time interaction. It is shown that the use of the Feynman approach to quantum theory in combination with the canonical approach allows one to extend quantum dynamics to describe the time evolution in the case of such interactions. In this way, using only the current concepts of quantum theory, a generalized equation of motion for state vectors is derived. In the case, where the fundamental interaction generating the dynamics in a system is local in time, this equation is equivalent to the Schrödinger equation. Explicit examples are given for an exactly solvable model. The proposed formalism is shown to provide a new insight into the problem of the description of nonlocal interactions in quantum field theory. It is shown that such a property of the equation of motion as nonlocality in time may be important for describing hadron-hadron interactions at low and intermediate energies.
\end{abstract}

\section{Introduction}

Various physical applications of quantum mechanics and quantum field theory require solutions of evolution problems. In standard canonical quantum theory, it is postulated that quantum dynamics is governed by the Schrödinger equation. However, as is well known, in quantum electrodynamics (QED) the ultraviolet divergences can be removed from the $\mathrm{S}$ matrix, but cannot be removed from quantities characterising the time evolution of quantum systems, since regularisation of the scattering matrix leads to a situation in which divergent terms automatically appear in the Schrödinger equation [1]. 
theory. This leads, in particular, to difficulties in finding a consistent QED description of natural broadening of spectral line profiles in atomic system [2]. Since locality has been argued to be the main cause of infinities in quantum field theory, it seems natural to resolve this problem by introducing a nonlocal form factor into the interaction Hamiltonian density. However, as is well known, such an introduction of a nonlocal form factor results in a loss of covariance. The reason for this is quite obvious. The Schrödinger equation is local in time, and the interaction Hamiltonian describes an instantaneous interaction. In nonrelativistic quantum mechanics processes of instantaneous interaction may be nonlocal in space. But in relativistic quantum theory a local in time process must be also local in space. Thus, for the introduction of nonlocality in the theory to be intrinsic consistent, one has to find a way of solving the evolution problem in the case when the dynamics in a system is generated by a nonlocal in time interaction. The solution of this problem may be important also for describing the low-energy hadron-hadron interaction. Indeed, nonlocality of interaction leads to the energy dependence of effective interaction operators. In recent years the possibility of using energy-dependent potentials to describe hadron-hadron interaction at low and intermediate energies has been widely discussed [3-10]. Interest in studying potentials of this type is provoked by still existing discrepancy between theory and experiment. For example, most "realistic" nucleon-nucleon (NN) potentials are not sufficiently strong to reproduce the observed ${ }^{3} \mathrm{H}$ and ${ }^{3} \mathrm{He}$ binding energies (see, for example, Ref.[11]). Moreover, the energy dependence of effective operators of hadron-hadron interaction is associated with the quark degrees of freedom, which are not included explicitly in the description of low-energy hadron interaction, and is predicted, for example, by quark models $[3,10]$. However, the dynamics of such systems cannot be described in the framework of the Hamiltonian formalism. Indeed, the energy dependence of the interaction Hamiltonian means that the total Hamiltonian also depends on energy, i.e. on its spectral parameter. Such an "operator" is not an operator in a rigorous sense. Hence the energy-dependent interaction operator cannot be interpreted as an interaction Hamiltonian.

Let us now turn to the Feynman formulation of quantum theory $[12,13]$. The main idea of this formulation is that quantum dynamics can be described without resorting to the Schrödinger equation. Feynman's theory starts with an analysis of the phenomenon of quantum interference which leads directly to the concept of the superposition of probability amplitudes. According to this concept, the probability amplitude of an event which can happen in several different wavs is a sum of probability amplitudes for each of these wav 
[12]. The Feynman formulation contains also as its essential idea the concept of a probability amplitude associated with a completely specified motion or path in space-time, and it is postulated that this probability amplitude has a phase proportional to the action, computed classically, for the corresponding path. Using this postulate together with the above assumption concerning the calculation of probabilities in quantum mechanics leads to Feynman's sum-over-paths formalism.

The theory of Feynman differs profoundly in its formulation from canonical quantum theory. These dissimilar approaches were proved to be equivalent and to complement one another in solving various problems in quantum physics. In the present paper we show that these two approaches can be used in combination for describing the time evolution of quantum systems. In this way an equation of motion for state vectors is derived. Being equivalent to the Schrödinger equation in the case of local interaction, this equation makes it possible to solve the evolution problem in the case where the interaction generating the dynamics in a quantum system is nonlocal in time.

We start with the evolution equation $\left|\psi(t)>=U\left(t, t_{0}\right)\right| \psi\left(t_{0}\right)>$, where $\mid \psi(t)>$ is a state vector and $U\left(t, t_{0}\right)$ is the evolution operator. Then, using the basic assumption of the Feynman formulation, we represent the matrix elements of the evolution operator as a sum of contributions from all alternative ways of realisation of the corresponding evolution process. The history of a quantum system is represented by some version of the time evolution of the system associated with completely specified instants of the beginning and end of the interaction in the system. In this way we get an expression for the matrix elements of the operator $U\left(t, t_{0}\right)$ in terms of probability amplitudes associated with such versions. An equation for these amplitudes is then derived from the requirement of unitarity for the evolution operator. It is shown that this equation can be regarded as an equation of motion. The concept of a generalized interaction operator is introduced. This operator is a generalization of the interaction Hamiltonian and generates the dynamics of a quantum system. In particular, the generalized interaction operator can be chosen so that the dynamics generated by this operator proves equivalent to the dynamics governed by the Schrödinger equation. At the same time, the equation of motion derived in this paper permits the generalization to the case where this equation manifests itself as a nonlocal in time dynamical equation. This point is illustrated in detail on an exactly solvable model. Finally, we discuss applications of the proposed formalism. This formalism is shown to provide a new insight into the problem of the description of nonlocal interactions in quantum field theorv. It is shown also that such a propertv of 
the proposed dynamical equation as nonlocality in time may be important for describing hadron-hadron interactions at low and intermediate energies.

\section{Basic Assumptions Of Quantum Theory}

We will assume the following properties for the states of quantum systems.

(i) The physical state of a system is represented by a vector (properly by a ray) of a Hilbert space.

(ii) An observable $\mathrm{A}$ is represented by a Hermitian hypermaximal operator $\alpha$. The eigenvalues $a_{r}$ of $\alpha$ give the possible values of A. An eigenvector $\mid \varphi_{r}^{(s)}>$ corresponding to the eigenvalue $a_{r}$ represents a state in which A has the value $a_{r}$. If the system is in the state $|\psi\rangle$, the probability $P_{r}$ of finding the value $a_{r}$ for $\mathrm{A}$, when a measurement is performed, is given by

$$
P_{r}=<\psi\left|P_{V_{r}}\right| \psi>=\sum_{s}\left|<\varphi_{r}^{(s)}\right| \psi>\left.\right|^{2},
$$

where $P_{V_{r}}$ is the projection operator on the eigenmanifold $V_{r}$ corresponding to $a_{r}$, and the sum $\Sigma_{s}$ is taken over a complete orthonormal set $\left|\varphi_{r}^{(s)}\right\rangle(\mathrm{s}=1,2, \ldots)$ of $V_{r}$. The state of the system immediately after the observation is described by the vector $P_{V_{r}} \mid \psi>$.

These assumptions are the main assumptions on which quantum theory is founded. In the canonical formalism these postulates are used together with the assumption that the time evolution of a state vector is governed by the Schrödinger equation. In our study we will not use this assumption. Instead the following postulate will be used:

(iii) The probability of an event is the absolute square of a complex number called the probability amplitude. The joint probability amplitude of a time-ordered sequence of events is product of the separate probability amplitudes of each of these events. The probability amplitude of an event which can happen in several different ways is a sum of the probability amplitudes for each of these ways.

The statements of the assumption (iii) express the well-known law for the quantum-mechanical probabilities. Within the canonical formalism this law is derived as one of the consequences of the theory. However, in the Feynman formulation of quantum theory this law is directly derived starting from the analysis of the phenomenon of quantum interference, and is used as a basic postulate of the theory. We will also use the following assumption:

(iv) Under space-time translations $x \rightarrow x+a$, the eigenstates $\mid n>$ of a system of non-interacting particles corresponding to the total momentum of 
follows:

$$
\left|n>\underset{x \rightarrow x+a}{\rightarrow} \exp \left(-i P_{n} a\right)\right| n>,
$$

where $E_{n}=\sum_{i} \sqrt{\mathbf{p}_{i}^{2}+m_{i}^{2}}\left(m_{i}\right.$ are the masses of the particles), $P_{n} a \equiv P_{n}^{\mu} a_{\mu}$, and $a$ is an arbitrary 4 -vector of displacement, and $n$ stands for the entire set of discrete and continuous variables that characterise the system in full.

Here and below, we use units where $\hbar=1$. The statement of the assumption (iv) expresses the well-known law of transformation of vectors describing states of non-interacting particles under space-time translations. This law is used, for example, as one of the starting points in constructing the axiomatic approaches to quantum field theory (see, for example, Refs.[14,15]).

The assumptions (i)-(iv) represent the current concepts of quantum theory. In the present paper we suggest a new way of using these concepts. In this way an equation of motion more general than the Schrödinger equation will be derived as a consequence of the assumptions (i)-(iv) and the requirement of conservation of probabilities.

\section{Time-Evolution Operator}

Let $H_{0}$ be the free Hamiltonian, i.e. the operator of the total energy of a system of particles travelling freely without interaction or external disturbance. The vectors $\mid n>$ are the eigenvectors of this operator: $H_{0}\left|n>=E_{n}\right| n>$. As is well known, from the assumption (ii) it follows that eigenvectors of any observable form a complete set of basis vectors in the Hilbert space. However, such continuum state vectors as the eigenvectors $\mid n>$ of the free Hamiltonian $H_{0}$ do not belong to the Hilbert space of the states. Following the Dirac formalism we will assume $\mid n>$ to be generalized basis vectors orthonormalized in a continuum (by using delta functions) in terms of which any vector of the Hilbert space can be expanded:

$$
\left|\psi>=\sum_{n}\right| n><n \mid \psi>
$$

From this and the assumption (iv) it follows that if there is no interaction in the system then any state vector has the following time-dependence:

$$
\left|\psi(t)>=\exp \left(-i H_{0} t\right)\right| \psi(0)>\text {. }
$$

Below we will employ the interaction representation in which the state vectors relate to the state vectors in the Schrödinger representation as follows: $\left|\psi(t)>_{I}=\exp \left(i H_{0} t\right)\right| \psi(t)>_{S}$. From (2) it follows that in the interaction 
time. Let us consider the probability amplitude $<\psi_{2}\left|U\left(t, t_{0}\right)\right| \psi_{1}>$ of finding, for a measurement at time $t$, the quantum system in the state $\mid \psi_{2}>$ if at time $t_{0}$ it was in the state $\left|\psi_{1}\right\rangle$. As it follows from the assumption (ii), this probability is the absolute square of the probability amplitude which is given by the scalar product $\left\langle\psi_{2} \mid \psi(t)\right\rangle$. Here $|\psi(t)\rangle=U\left(t, t_{0}\right)\left|\psi_{1}\right\rangle$ is the state at time $t$ to which the system evolves from the state $\left|\psi_{1}\right\rangle$ at time $t_{0}$ if it is not disturbed by measurements; accordingly, $U\left(t, t_{0}\right)$ is the operator describing such an evolution. In the canonical approach to quantum theory $U\left(t, t_{0}\right)$ is postulated to be a unitary operator

$$
U^{+}\left(t_{2}, t_{1}\right) U\left(t_{2}, t_{1}\right)=U\left(t_{2}, t_{1}\right) U^{+}\left(t_{2}, t_{1}\right)=\mathbf{1},
$$

with the group property

$$
U\left(t_{2}, t_{1}\right) U\left(t_{1}, t_{0}\right)=U\left(t_{2}, t_{0}\right), \quad U\left(t_{0}, t_{0}\right)=\mathbf{1} .
$$

In the case of an isolated system (only such systems will be considered in the present paper), the evolution operator in the Schrödinger picture $U_{s}\left(t_{2}, t_{1}\right) \equiv \exp \left(-i H_{0} t_{2}\right) U\left(t_{2}, t_{1}\right) \exp \left(i H_{0} t_{1}\right)$ depends on the difference $\left(t_{2}-t_{1}\right)$ only, so that the operators $V(t) \equiv U_{s}(t, 0)$ constitute a one-parameter group of unitary operators, with the group property

$$
V\left(t_{1}+t_{2}\right)=V\left(t_{1}\right) V\left(t_{2}\right), \quad V(0)=0 .
$$

If these operators are assumed to be strongly continuous, i.e. if

$$
\lim _{t_{2} \rightarrow t_{1}}\left\|V\left(t_{2}\right)\left|\psi>-V\left(t_{1}\right)\right| \psi>\right\|=0
$$

then from Stone's theorem it follows [16] that this one-parameter group has a self-adjoint infinitesimal generator $H$ :

$$
V(t)=\exp (-i H t), \quad i d / d t V(t)=H V(t) .
$$

Identifying $H$ with the total Hamiltonian as usual, we get the time-dependent Schrödinger equation: $i \frac{d \mid \psi_{s}(t)>}{d t}=H \mid \psi_{s}(t)>$, where $\left|\psi_{s}(t)>=V(t)\right| \psi_{s}(t=0)>$ . However, the condition (6) seems to be too strong. From the physical point of view, it is enough to require that

$$
<\psi_{2}\left|V\left(t_{2}\right)\right| \psi_{1}>\underset{t_{2} \rightarrow t_{1}}{\rightarrow}<\psi_{2}\left|V\left(t_{1}\right)\right| \psi_{1}>
$$

for any physically realisable states $\left|\psi_{1}\right\rangle$ and $\left|\psi_{2}\right\rangle$ [14]. Note in this connection that there are normalized vectors in the Hilbert space that represent the states for which energy of a system is infinite. Such states cannot 
elements of the evolution operator need not be continuous. For this reason, in the present paper we will not restrict ourselves to the strongly continuous evolution operators. We will only assume that the evolution operator satisfies the condition (7). This means that we will not use the assumption that the time evolution of a state vector is governed by the Schrödinger equation as the basic dynamical postulate.

According to the assumption (iii), the probability amplitude of an event which can happen in several different ways is a sum of contributions from each alternative way. In particular, the amplitude $<\psi_{2}\left|U\left(t, t_{0}\right)\right| \psi_{1}>$ can be represented as a sum of contributions from all alternative ways of realisation of the corresponding evolution process. Dividing these alternatives in different classes, we can then analyse such a probability amplitude in different ways [13]. For example, subprocesses with definite instants of the beginning and end of the interaction in the system can be considered as such alternatives. Let $<n_{2}\left|U\left(t, t_{0} ; t_{2}, t_{1}\right)\right| n_{1}>$ be the probability amplitude that if at time $t_{0}$ the system was in the state $\left|n_{1}\right\rangle$, then the interaction in the system will begin at time $t_{1}$ and end at time $t_{2}$, and at time $t$ the system will be in the state $\mid n_{2}>$. According to the postulate (iii), the amplitudes $<n_{2}\left|U\left(t, t_{0} ; t_{2}, t_{1}\right)\right| n_{1}>$ determine the contributions from the above alternatives, and $<n_{2}\left|U\left(t, t_{0}\right)\right| n_{1}>$ can be represented as a sum (more precisely as an integral) of these contributions

$$
<n_{2}\left|U\left(t, t_{0}\right)\right| n_{1}>=\varphi\left(n_{2}, n_{1}, t, t_{0}\right)+\int_{t_{0}}^{t} d t_{2} \int_{t_{0}}^{t_{2}} d t_{1}<n_{2}\left|U\left(t, t_{0} ; t_{2}, t_{1}\right)\right| n_{1}>,
$$

where $\varphi\left(n_{2}, n_{1}, t, t_{0}\right)$ is the probability amplitude that if at time $t_{0}$ the system was in the state $\left|n_{1}\right\rangle$, then the particles of the system will not interact in the time interval $\left(t_{0}, t\right)$, and at time $t$ the system will be in the state $\left|n_{2}\right\rangle$. Thus the first term on the right-hand side of (8) is the contribution from the alternative subprocess in the case of which the particles of the system do not interact. From the postulate (iii), it follows that the probability amplitude $<n_{2}\left|U\left(t, t_{0} ; t_{2}, t_{1}\right)\right| n_{1}>$ is expressible as a product

$$
<n_{2}\left|U\left(t, t_{0} ; t_{2}, t_{1}\right)\right| n_{1}>=\sum_{n^{\prime}} \sum_{n} \varphi\left(n_{2}, n^{\prime}, t, t_{2}\right)<n^{\prime}\left|\tilde{S}\left(t_{2}, t_{1}\right)\right| n>\varphi\left(n, n_{1}, t_{1}, t_{0}\right),
$$

where $<n_{2}\left|\tilde{S}\left(t_{2}, t_{1}\right)\right| n_{1}>$ is the probability amplitude that if at time $t_{1}$ the system was in the state $\left|n_{1}\right\rangle$, then the interaction in the system will begin at time $t_{1}$ and will end at time $t_{2}$, and at this time the system will be in the state $\mid n_{2}>$.

The evolution operator $U\left(t, t_{0}\right)$ has a natural decomposition 
Here the unit operator represents the no-interaction part; its matrix elements are delta functions which make the final momenta the same as the initial momenta. The operator $R\left(t, t_{0}\right)$ represents the interaction part. From (8) and (10) it follows that $\varphi\left(n_{2}, n_{1}, t_{2}, t_{1}\right)=<n_{2} \mid n_{1}>$, and hence $<n_{2}\left|U\left(t, t_{0} ; t_{2}, t_{1}\right)\right| n_{1}>=<n_{2}\left|\tilde{S}\left(t_{2}, t_{1}\right)\right| n_{1}>$. Thus equation (8) can be rewritten in the form

$$
<n_{2}\left|U\left(t, t_{0}\right)\right| n_{1}>=<n_{2}\left|n_{1}>+\int_{t_{0}}^{t} d t_{2} \int_{t_{0}}^{t_{2}} d t_{1}<n_{2}\right| \tilde{S}\left(t_{2}, t_{1}\right) \mid n_{1}>
$$

accordingly, the evolution operator $U\left(t, t_{0}\right)$ can be expressed in terms of the operators $\tilde{S}\left(t_{2}, t_{1}\right)$ whose matrix elements are the amplitudes $<n_{2}\left|\tilde{S}\left(t_{2}, t_{1}\right)\right| n_{1}>$ :

$$
U\left(t, t_{0}\right)=\mathbf{1}+\int_{t_{0}}^{t} d t_{2} \int_{t_{0}}^{t_{2}} d t_{1} \tilde{S}\left(t_{2}, t_{1}\right)
$$

Equation (12) defines the evolution operator $U\left(t_{2}, t_{1}\right)$ only for $t_{2} \geq t_{1}$. Using (3), the evolution operator $U\left(t_{2}, t_{1}\right)$ for $t_{2}<t_{1}$ can be constructed as follows:

$$
U\left(t_{2}, t_{1}\right)=U^{-1}\left(t_{1}, t_{2}\right)=U^{+}\left(t_{1}, t_{2}\right) .
$$

It should be noted that in general $\tilde{S}\left(t_{2}, t_{1}\right)$ may be an operator-valued distribution, since only $U\left(t, t_{0}\right)$ has to be an operator on the Hilbert space. Nevertheless, throughout this paper we will use term "operator" for $\tilde{S}\left(t_{2}, t_{1}\right)$.

To clarify the role which the operator $\tilde{S}\left(t_{2}, t_{1}\right)$ plays in the proposed formalism, note the following. The Feynman formulation is based on the assumption that the history of a system can be represented by some path in space-time. From the postulate (iii) it then follows that the probability amplitudes of any event is a sum of the probability amplitudes that a particle has a completely specified path in space-time. The contribution from a single path is postulated to be an exponential whose (imaginary) phase is the classical action (in units of $\hbar$ ) for the path in question. In the proposed formalism the history of a system is represented by the version of the time evolution of the system associated with completely specified instants of the beginning and end of the interaction in the system. Such a description of the history of a system is more general and requires no supplementary postulates like the above assumptions of the Feynman formulation. On the other hand, the probability amplitudes $<\psi_{2}\left|\tilde{S}\left(t_{2}, t_{1}\right)\right| \psi_{1}>$ in terms of which we describe quantum dynamics are used in the spirit of Feynman's theory: The probability amplitude of any event is represented as a sum of these amplitudes. Bellow we will show that the requirement of unitarity for the evolution operator given by (12) leads to an equation for the operator $\tilde{S}\left(t_{2}, t_{1}\right)$ which can be regarded as 
As we have noted, $<\psi\left(t_{2}\right)\left|\tilde{S}\left(t_{2}, t_{1}\right)\right| \psi\left(t_{1}\right)>$ is the probability amplitude that if at time $t_{1}$ the system was in the state $\left|\psi_{1}\right\rangle$, then the interaction in the system will begin at time $t_{1}$ and will end at time $t_{2}$, and at this time the system will be found to be in the state $\left|\psi\left(t_{2}\right)\right\rangle$. Here this probability amplitude is represented by the matrix element $<\psi\left(t_{2}\right)\left|\tilde{S}\left(t_{2}, t_{1}\right)\right| \psi\left(t_{1}\right)>$ in the interaction picture. However, the same probability amplitude can be represented by the matrix element $<\psi_{s}\left(t_{2}\right)\left|\tilde{S}_{s}\left(t_{2}, t_{1}\right)\right| \psi_{s}\left(t_{1}\right)>$ in the Schrödinger picture, where $\tilde{S}_{s}\left(t_{2}, t_{1}\right)$ is the operator describing the transformation of a state at time $t_{1}$ into the state at time $t_{2}$ caused by the interaction in the system that begins at time $t_{1}$ and ends at time $t_{2}$. Being an operator in the Schrödinger picture, $\tilde{S}_{s}\left(t_{2}, t_{1}\right)$ depends on the difference $\left(t_{2}-t_{1}\right)$ only: $\tilde{S}_{s}\left(t_{2}, t_{1}\right) \equiv \tilde{T}\left(t_{2}-t_{1}\right)$. Since $<\psi_{s}\left(t_{2}\right)\left|\tilde{S}_{s}\left(t_{2}, t_{1}\right)\right| \psi_{s}\left(t_{1}\right)>$ and $<\psi_{s}\left(t_{2}\right)\left|\tilde{T}\left(t_{2}-t_{1}\right)\right| \psi_{s}\left(t_{1}\right)>$ represent the same probability amplitude, we have

$$
<\psi_{s}\left(t_{2}\right)\left|\tilde{S}_{s}\left(t_{2}, t_{1}\right)\right| \psi_{s}\left(t_{1}\right)>=<\psi_{s}\left(t_{2}\right)\left|\tilde{T}\left(t_{2}-t_{1}\right)\right| \psi_{s}\left(t_{1}\right)>.
$$

Taking into account the relation between the states in the Schrödinger and interaction pictures given by $|\psi(t)\rangle=\exp \left(i H_{0} t\right)\left|\psi_{s}(t)\right\rangle$, from (14), we get

$$
\tilde{S}\left(t_{2}, t_{1}\right)=\exp \left(i H_{0} t_{2}\right) \tilde{T}\left(t_{2}-t_{1}\right) \exp \left(-i H_{0} t_{1}\right)>.
$$

Let us now consider the scattering matrix. Letting $t_{0} \rightarrow-\infty$ and $t \rightarrow \infty$ in (12), we get the $\mathrm{S}$ matrix in the following form:

$$
<n_{2}|S| n_{1}>=<n_{2}\left|n_{1}>+\int_{-\infty}^{\infty} d t_{2} \int_{-\infty}^{t_{2}} d t_{1}<n_{2}\right| \tilde{S}\left(t_{2}, t_{1}\right) \mid n_{1}>.
$$

By using (15), this expression can be rewritten in the form

$$
<n_{2}|S| n_{1}>=<n_{2}\left|n_{1}>-2 \pi i \delta\left(E_{n_{2}}-E_{n_{1}}\right)<n_{2}\right| T\left(E_{n_{1}}\right) \mid n_{1}>,
$$

where the operator $T(z)$ is defined by

$$
<n_{2}|T(z)| n_{1}>=i \int_{-\infty}^{\infty} d \tau \exp (i z \tau)<n_{2}|F(\tau)| n_{1}>,
$$

with

$$
<n_{2}|F(\tau)| n_{1}>=<n_{2}|\tilde{T}(\tau)| n_{1}>, \quad \tau \geq 0,
$$

and $<n_{2}|F(\tau)| n_{1}>=0 \quad$ for $\quad \tau<0$. The fact that $F(\tau)=0$ for $\tau<0$ can be considered as an expression of causality, since $\tau$ is assumed to be the duration time of interaction that has to be positive. A remarkable consequence of this fact is that $<n_{2}|T(z)| n_{1}>$ is analytic in the upper half of the complex $z$ plane. 


\section{Unitarity Condition and its Consequences}

Let us consider the unitarity condition that expresses the principle of conservation of probabilities. Substituting (10) into the unitarity condition (3) gives

$$
\begin{aligned}
& <n_{2}\left|R\left(t, t_{0}\right)\right| n_{1}>-<n_{2}\left|R^{+}\left(t, t_{0}\right)\right| n_{1}>= \\
& =i \sum_{n}<n_{2}\left|R^{+}\left(t, t_{0}\right)\right| n><n\left|R\left(t, t_{0}\right)\right| n_{1}>, \\
& <n_{2}\left|R\left(t, t_{0}\right)\right| n_{1}>-<n_{2}\left|R^{+}\left(t, t_{0}\right)\right| n_{1}>= \\
& =i \sum_{n}<n_{2}\left|R\left(t, t_{0}\right)\right| n><n\left|R^{+}\left(t, t_{0}\right)\right| n_{1}>.
\end{aligned}
$$

From $(10),(12)$ and (15) it follows that the operator $R\left(t, t_{0}\right)$ can be written as

$$
R\left(t, t_{0}\right)=-i \int_{t_{0}}^{t} d t_{2} \int_{t_{0}}^{t_{2}} d t_{1} \exp \left(i H_{0} t_{2}\right) \tilde{T}\left(t_{2}-t_{1}\right) \exp \left(-i H_{0} t_{1}\right) .
$$

Taking into account that, according to (18), the operator $\tilde{T}(\tau)$ can be represented in the form

$$
\tilde{T}(\tau)=-\frac{i}{2 \pi} \int_{-\infty}^{\infty} d x \exp (-i z \tau) T(z)
$$

where $z=x+i y, x$ and $y$ are real, and $y>0$, we get

$$
\begin{gathered}
<n_{2}\left|R\left(t, t_{0}\right)\right| n_{1}>=-\frac{1}{2 \pi} \int_{t_{0}}^{t} d t_{2} \int_{t_{0}}^{t_{2}} d t_{1} \int_{-\infty}^{\infty} d x \\
\times \exp \left(i E_{n_{2}} t_{2}\right) \exp \left[-i z\left(t_{2}-t_{1}\right)\right] \exp \left(-i E_{n_{1}} t_{1}\right)<n_{2}|T(z)| n_{1}>.
\end{gathered}
$$

Let us assume that $<n_{2}|T(z)| n_{1}>$ satisfies the condition

$$
\lim _{|z| \rightarrow \infty} \frac{<n_{2}|T(z)| n_{1}>}{z}=0 .
$$

Note in this connection that the properties of $<n_{2}|T(z)| n_{1}>$ at infinity depend on the behaviour of $<n_{2}\left|\tilde{S}\left(t_{2}, t_{1}\right)\right| n_{1}>$ in the limit $t_{2} \rightarrow t_{1}$. As will be shown bellow, in the proposed formalism this behaviour determines the dynamics in the system. Thus, by assuming that $<n_{2}|T(z)| n_{1}>$ satisfies the condition (25), we restrict ourselves to considering some class of the dynamical schemes. However, the important thing for us is that, as it will be shown below, the Hamiltonian dynamics belongs to this class. Taking into account the condition (25), from (24), we get (see Appendix A)

$$
<n_{2}\left|R\left(t, t_{0}\right)\right| n_{1}>=
$$

$1 \rho^{\infty}, \exp \left[-i\left(z-E_{n_{2}}\right) t\right] \exp \left[i\left(z-E_{n_{1}}\right) t_{0}\right]$ 
Substituting of (26) into (20) gives

$$
\begin{gathered}
\int_{-\infty}^{\infty} d x_{1} \frac{\exp \left[-i\left(z_{1}-E_{n_{2}}\right) t\right] \exp \left[i\left(z_{1}-E_{n_{1}}\right) t_{0}\right]}{\left(z_{1}-E_{n_{2}}\right)\left(z_{1}-E_{n_{1}}\right)}<n_{2}\left|T\left(z_{1}\right)\right| n_{1}>- \\
-\int_{-\infty}^{\infty} d x_{2} \frac{\exp \left[i\left(z_{2}^{*}-E_{n_{1}}\right) t\right] \exp \left[-i\left(z_{2}^{*}-E_{n_{2}}\right) t_{0}\right]}{\left(z_{2}^{*}-E_{n_{2}}\right)\left(z_{2}^{*}-E_{n_{1}}\right)}<n_{2}\left|T^{+}\left(z_{2}\right)\right| n_{1}>= \\
=<n_{2}\left|M\left(t, t_{0}\right)\right| n_{1}>,
\end{gathered}
$$

where $z_{1}=x_{1}+i y, z_{2}=x_{2}+i y, y>0$, and

$$
\begin{gathered}
<n_{2}\left|M\left(t, t_{0}\right)\right| n_{1}>=\frac{i}{2 \pi} \int_{-\infty}^{\infty} d x_{1} \int_{-\infty}^{\infty} d x_{2} \\
\times \frac{\exp \left[i\left(z_{2}^{*}-z_{1}\right)\left(t-t_{0}\right)\right] \exp \left[i\left(E_{n_{2}}-E_{n_{1}}\right) t_{0}\right]}{\left(z_{2}^{*}-E_{n_{2}}\right)\left(z_{1}-E_{n_{1}}\right)}<n_{2}\left|B\left(z_{2}, z_{1}\right)\right| n_{1}>,
\end{gathered}
$$

with

$$
<n_{2}\left|B\left(z_{2}, z_{1}\right)\right| n_{1}>=\sum_{n} \frac{<n_{2}\left|T^{+}\left(z_{2}\right)\right| n><n\left|T\left(z_{1}\right)\right| n_{1}>}{\left(z_{2}^{*}-E_{n}\right)\left(z_{1}-E_{n}\right)} .
$$

Note that equation (27) derived in this way must be satisfied for $t \geq t_{0}$. However, taking into account the analytic properties of $\left\langle n_{2}|T(z)| n_{1}\right\rangle$, one can easily verify that both sides of $(27)$ are equal to zero for $t<t_{0}$. Thus equation (27) is valid for all $t$ and $t_{0}$. Represent the operator $B\left(z_{1}, z_{2}\right)$ in the form

$$
B\left(z_{1}, z_{2}\right)=\frac{T\left(z_{1}\right)-T^{+}\left(z_{2}\right)}{z_{2}^{*}-z_{1}}+F\left(z_{1}, z_{2}\right)
$$

Substituting (30) into (27) and taking into account the analytic properties of $<n_{2}|T(z)| n_{1}>$, we have then

$$
\begin{gathered}
\int_{-\infty}^{\infty} d x_{1} \int_{-\infty}^{\infty} d x_{2} \frac{\exp \left[i\left(z_{2}^{*}-z_{1}\right)\left(t-t_{0}\right)\right] \exp \left[i\left(E_{n_{2}}-E_{n_{1}}\right) t_{0}\right]}{\left(z_{2}^{*}-E_{n_{2}}\right)\left(z_{1}-E_{n_{1}}\right)} \\
\times<n_{2}\left|F\left(z_{1}, z_{2}\right)\right| n_{1}>=0 .
\end{gathered}
$$

Since equation (31) must be satisfied for all $t$ and $t_{0}$, we get

$$
<n_{2}\left|F\left(z_{1}, z_{2}\right)\right| n_{1}>=0
$$

From this it follows that

$$
T\left(z_{1}\right)-T^{+}\left(z_{2}\right)=\left(z_{2}^{*}-z_{1}\right) \sum_{n} \frac{T^{+}\left(z_{2}\right)|n><n| T\left(z_{1}\right)}{\left(z_{2}^{*}-E_{n}\right)\left(z_{1}-E_{n}\right)} .
$$

Proceeding in an analogous way, from (21), we obtain

$$
T\left(z_{1}\right)|n><n| T^{+}\left(z_{2}\right)
$$


It is easy to show then that the following equation results from (33) and (34):

$$
T\left(z_{1}\right)-T\left(z_{2}\right)=\left(z_{2}-z_{1}\right) \sum_{n} \frac{T\left(z_{2}\right)|n><n| T\left(z_{1}\right)}{\left(z_{2}-E_{n}\right)\left(z_{1}-E_{n}\right)} .
$$

Thus we have shown that for the evolution operator given by (12) to be unitary the operator $T(z)$ must satisfy equations (33), (34) and (35). At the same time, as it shown in Appendix B, the evolution operator given by (12) satisfies the composition law (4), provided equation (35) is valid. Note that equation (35) can be derived from the composition law (4) and the representation (12) in the same way in which (33) has been derived from this representation and the unitarity condition (20). However, equations (33) and (34) are more general than equation (35). In fact, (35) results from (33) and (34). But for (33) and (34) to result from (35) the additional assumption that $T^{+}(z)=T\left(z^{*}\right)$ is required.

Equation (35) plays a key role in the proposed formalism. It has been derived as a consequence of such fundamental physical principles of quantum theory as the requirement of conservation of probabilities and the principle of the superposition of probability amplitudes. It should be noted that an equation having the same form as equation (35) has been derived for the scattering $\mathrm{T}$ matrix as a consequence of the above principles within the approach to the scattering theory developed in Refs.[17-20]. That equation was derived as an off-shell generalization of the unitarity condition for the scattering S matrix, and may be regarded as a particular case of equation (35).

\section{Equation of Motion for State Vectors}

In the previous section we have derived equations (33), (34) and (35) for the operator $T(z)$. It now becomes necessary for us to obtain an equation directly for the operator $\tilde{S}\left(t_{2}, t_{1}\right)$ which is of central importance in our formalism. It can be shown (see Appendix C) that for equation (35) to be valid for any $z_{1}$ and $z_{2}$ the operator must satisfy the following equations:

$$
\begin{gathered}
\left(1-\exp \left[i a\left(t_{2}-t_{1}\right)\right]\right) \tilde{S}\left(t_{2}, t_{1}\right)= \\
=\int_{t_{1}}^{t_{2}} d t_{4} \int_{t_{1}}^{t_{4}} d t_{3} \exp \left[i a\left(t_{2}-t_{4}\right)\right]\left(1-\exp \left[i a\left(t_{4}-t_{3}\right)\right]\right) \tilde{S}\left(t_{2}, t_{4}\right) \tilde{S}\left(t_{3}, t_{1}\right), \\
\left(t_{2}-t_{1}\right) \tilde{S}\left(t_{2}, t_{1}\right)=\int_{t_{1}}^{t_{2}} d t_{4} \int_{t_{1}}^{t_{4}} d t_{3}\left(t_{4}-t_{3}\right) \tilde{S}\left(t_{2}, t_{4}\right) \tilde{S}\left(t_{3}, t_{1}\right),
\end{gathered}
$$

where $a=z_{2}-z_{1}$. Substituting (15) into (37), we obtain then the corresponding equation for the operator $\tilde{T}(\tau)$ 
It should be noted that (38) coincides in form with the equation for the operator $\tilde{T}(\tau)$ derived within scattering theory [21]. However, (38) was initially considered as an equation allowing one to find out what boundary conditions for equation (35) are admissible. In Ref.[22] it was shown that this equation enables one to construct a new class models in nonrelativistic quantum scattering theory, which were shown to open new possibilities for describing hadron-hadron interactions at low energies.

As we will show below, equation (37) allows one to obtain the amplitudes $<n_{2}\left|\tilde{S}\left(t_{2}, t_{1}\right)\right| n_{1}>$ for any $t_{1}$ and $t_{2}$ if the amplitudes $<n_{2}\left|\tilde{S}\left(t_{2}^{\prime}, t_{1}^{\prime}\right)\right| n_{1}>$ corresponding to infinitesimal duration times $\tau=t_{2}^{\prime}-t_{1}^{\prime}$ of interaction are known. It is natural to assume that most of the contribution to the evolution operator in the limit $t_{2} \rightarrow t_{1}$ comes from the processes associated with an fundamental interaction in the system under study. Denoting this contribution by $H_{\text {int }}\left(t_{2}, t_{1}\right)$, the operator $\tilde{S}\left(t_{2}, t_{1}\right)$ can be represented in the form

$$
\tilde{S}\left(t_{2}, t_{1}\right)=H_{\text {int }}\left(t_{2}, t_{1}\right)+\tilde{S}_{1}\left(t_{2}, t_{1}\right),
$$

where $\tilde{S}_{1}\left(t_{2}, t_{1}\right)$ is the part of the operator $\tilde{S}\left(t_{2}, t_{1}\right)$ which in the limit $t_{2} \rightarrow t_{1}$ gives the negligibly small contribution to the evolution operator little in comparison with $H_{\text {int }}\left(t_{2}, t_{1}\right)$. We will assume that the operator $H_{\text {int }}\left(t_{2}, t_{1}\right)$ contain all the dynamical information that is needed to construct the evolution operator $U\left(t_{2}, t_{1}\right)$. Thus we will assume the operator $H_{\text {int }}\left(t_{2}, t_{1}\right)$ to play the role which the interaction Hamiltonian plays in the ordinary formulation of quantum theory: It generates dynamics in a system. This operator can be regarded as a generalization of the interaction Hamiltonian, and we will call it the generalized interaction operator. Obviously, the operator $H_{\text {int }}\left(t_{2}, t_{1}\right)$ must satisfy (37) in the limit $t_{2} \rightarrow t_{1}$

$$
F_{1}\left(t_{2}, t_{1}\right) \underset{t_{2} \rightarrow t_{1}}{\rightarrow} 0
$$

where

$$
F_{1}\left(t_{2}, t_{1}\right)=-\left(t_{2}-t_{1}\right) H_{\text {int }}\left(t_{2}, t_{1}\right)+\int_{t_{1}}^{t_{2}} d t_{4} \int_{t_{1}}^{t_{4}} d t_{3}\left(t_{4}-t_{3}\right) H_{\text {int }}\left(t_{2}, t_{4}\right) H_{\text {int }}\left(t_{3}, t_{1}\right) \text {. }
$$

According to (15), the operator $H_{\text {int }}\left(t_{2}, t_{1}\right)$ can be represented in the form

$$
H_{\text {int }}\left(t_{2}, t_{1}\right)=\exp \left(i H_{0} t_{2}\right) H_{\text {int }}^{(s)}\left(t_{2}-t_{1}\right) \exp \left(-i H_{0} t_{1}\right),
$$

where $H_{i n t}^{(s)}\left(t_{2}-t_{1}\right)$ is the generalized interaction operator in the Schrödinger picture. If $H_{\text {int }}\left(t_{2}, t_{1}\right)$ is specified, equation (37) allows one to find the operator $\tilde{S}\left(t_{2}, t_{1}\right)$. Formula $(12)$ can then be used to construct the evolution operator $U\left(t, t_{0}\right)$ and accordingly the state vector 
at any time $t$. Thus (37) can be regarded as an equation of motion for states of a quantum system.

From the mathematical point of view the requirement that $H_{\text {int }}\left(t_{2}, t_{1}\right)$ contains all the dynamic information that is needed for constructing $U\left(t_{2}, t_{1}\right)$ means that the operator $H_{\text {int }}\left(t_{2}, t_{1}\right)$ must have such a form that the equation (37) has a unique solution having the following behaviour near the point $t_{2}=t_{1}$ :

$$
\tilde{S}\left(t_{2}, t_{1}\right) \underset{t_{2} \rightarrow t_{1}}{\rightarrow} H_{\text {int }}\left(t_{2}, t_{1}\right)+o\left(\tau^{\epsilon}\right)
$$

where $\tau=t_{2}-t_{1}$ and the value of $\epsilon$ depends on the form of the operator $H_{\text {int }}\left(t_{2}, t_{1}\right)$. In order to clarify this point, note that equation (38) is equivalent to the following equation for the operator $T(z)$ given by (18):

$$
\frac{d T(z)}{d z}=T(z) G^{(2)}(z) T(z)
$$

where

$$
G^{(2)}(z)=-\sum_{n} \frac{|n><n|}{\left(z-E_{n}\right)^{2}} .
$$

The correspondence between equation (38) and the differential equation (44) can be easily stated in the same way in which we have stated correspondence between equations (35) and (36). Thus, instead of solving (37) or (38), one can solve equation (44) for the operator $T(z)$. The operator $\tilde{T}(\tau)$ and correspondingly the operator $\tilde{S}\left(t_{2}, t_{1}\right)$ can then be obtained by using $(23)$. At the same time, according to $(26)$, the operator $T(z)$ can be dirrectly used for constructing the evolution operator. According to (18), (39) and (41), the operator $T(z)$ has the following asymptotic behaviour for $|z| \rightarrow \infty$ :

$$
T(z) \underset{|z| \rightarrow \infty}{\rightarrow} B(z)+o\left(|z|^{-\beta}\right)
$$

where

$$
B(z)=i \int_{0}^{\infty} d \tau \exp (i z \tau) H_{\text {int }}^{(s)}(\tau)
$$

and $\beta=1+\epsilon$. From (44) and (45) it follows that the operator $B(z)$ must satisfy the following asymptotic condition:

$$
\frac{d B(z)}{d z} \underset{|z| \rightarrow \infty}{\rightarrow} B(z) G^{(2)}(z) B(z)+o\left(|z|^{-\beta}\right) .
$$

The above requirements, which the operator $H_{i n t}^{(s)}(\tau)$ has to meet, mean that $B(z)$ must be so close to the solution of equation (44) in the limit $|z| \rightarrow \infty$ that this differential equation has a unique solution having the asymptotic behaviour $(45)$. The operator $B(z)$ represents the contribution which $H_{i n t}^{(s)}(\tau)$ 
Let us now show that the Schrödinger equation results from equation (37) and hence the dynamics of a quantum system is equivalent to the Hamiltonian dynamics in the case when the generalized interaction operator is of the form

$$
H_{\text {int }}^{(s)}(\tau)=-2 i \delta(\tau) H_{I}
$$

$H_{I}$ being a self-adjoint operator. The delta function $\delta(\tau)$ in (48) emphasises the fact that in this case, the fundamental interaction is instantaneous. As it is shown in Appendix $\mathrm{C}$, from (37) it follows that $\tilde{S}\left(t_{2}, t_{1}\right)$ must satisfy also equation (36) for any $a$. Letting $a \rightarrow i \infty$ in (36) and taking into account (48), we get the following equation:

$$
\begin{gathered}
<n_{2}\left|\tilde{S}\left(t_{2}, t_{1}\right)\right| n_{1}>=-2 i \delta\left(t_{2}-t_{1}\right)<n_{2}\left|H_{I}\left(t_{1}\right)\right| n_{1}> \\
\quad-i \sum_{n} \int_{t_{1}}^{t_{2}} d t_{3}<n_{2}\left|H_{I}\left(t_{2}\right)\right| n><n\left|\tilde{S}\left(t_{3}, t_{4}\right)\right| n_{1}>,
\end{gathered}
$$

where $H_{I}(t)=\exp \left(i H_{0} t\right) H_{I} \exp \left(-i H_{0} t\right)$. Solving this equation by expanding $\tilde{S}\left(t_{2}, t_{1}\right)$ in terms of $H_{I}(t)$, one can easily get

$$
\begin{gathered}
\tilde{S}\left(t, t_{0}\right)=-2 i \delta\left(t-t_{0}\right) H_{I}\left(t_{0}\right)+ \\
+\sum_{n=2}^{\infty}(-i)^{n} \int_{t_{0}}^{t} d t_{1} \int_{t_{0}}^{t_{1}} d t_{2} \cdots \int_{t_{0}}^{t_{n-3}} d t_{n-2} H_{I}(t) H_{I}\left(t_{1}\right) \cdots H_{I}\left(t_{n-2}\right) H_{I}\left(t_{0}\right) .
\end{gathered}
$$

Thus we have shown that for the case under study equation (37) allows one to obtain $\tilde{S}\left(t_{2}, t_{1}\right)$ for any $t_{1}$ and $t_{2}$ starting from the contribution to the evolution operator coming from the process associated with the instantaneous interaction.

Inserting (50) into (12) yields

$$
U\left(t, t_{0}\right)=\mathbf{1}+\sum_{n=1}^{\infty}(-i)^{n} \int_{t_{0}}^{t} d t_{1} \cdots \int_{t_{0}}^{t_{n-1}} d t_{n} H_{I}\left(t_{1}\right) H_{I}\left(t_{2}\right) \cdots H_{I}\left(t_{n}\right) .
$$

This expression coincides in form with the Dyson expansion of the evolution operator. From this it follows that the operator $H_{I}(t)$ has to be identified with the interaction Hamiltonian. As is well known, the series (51) is convergent, provided the operator $H_{I}(t)$ is bounded. This means that the series $(50)$ is also convergent in this case. On the other hand, substituting (49) into (12), we easily get the following equation for the evolution operator $U\left(t_{2}, t_{1}\right)$ :

$$
U\left(t_{2}, t_{1}\right)=\mathbf{1}-i \int_{t_{1}}^{t_{2}} d t H_{I}(t) U\left(t, t_{1}\right) .
$$

This equation is the integral form of the Schrödinger equation for the evolution operator 
Hence in the case when $H_{i n t}^{(s)}(\tau)$ is of the form (48), from (37) it follows that $\mid \psi(t)>$ given by (42) satisfies the Schrödinger equation

$$
\frac{d \mid \psi(t)>}{d t}=-i H_{I}(t) \mid \psi(t)>.
$$

Thus we have shown the equivalence of the dynamics governed by equation (37) to the Hamiltonian dynamics in the case when the generalized interaction

operator $H_{i n t}^{(s)}(\tau)$ is of the form (48). At the same time, as will be shown below, equation (37) permits the generalization to the case when the operator $H_{\text {int }}^{(s)}(\tau)$ has no such a singularity at the point $t_{2}=t_{1}$ as the delta function. In this case the fundamental interaction generating the dinamics in a quantum system is nonlocal in time: The evolution operator is defined by $H_{i n t}^{(s)}(\tau)$ as a function of the time duration $\tau$ of the interaction.

It should be noted that the concept of nonlocal in time potentials was first introduced within the optical-potential model. This concept, for example, is used in the theory of time-dependent optical potentials (see, for example, $[23,24]$ and references therein). The optical potentials are introduced in the case when only state vectors belonging some subspace of the Hilbert space are included explicitly in the description of the time evolution of a quantum system. Such potentials which account for the coupling between this subspace and its complementary part of the Hilbert space are nonlocal in time and, thus, depends on the history of a dynamical system. The nonlocal form of the optical potentials is an expression of the loss of probability from the above subspace. However, the optical-potential model is one of the methods for the description of the time evolution of quantum systems the dynamics of which is generated by the local in time interaction being described by the interaction Hamiltonian. In the present formalism such a dynamics corresponds to the particular case when the generalized interaction operator is of the form (48). At the same time our formalism permits the generalization to the case when the fundamental interaction generating the dynamics in a quantum system itself is nonlocal in time and hence the nonlocality of the interaction does not lead to the loss of probability in the system.

\section{Exactly Solvable Model}

Let us consider the evolution problem for two nonrelativistic particles in the c.m.s. We denote the relative momentum by $\mathbf{p}$ and the reduced mass by $\mu$. Assume that the generalized interaction operator in the Schrödinger picture 
$H_{\text {int }}^{(s)}(\tau)$ has the form

$$
<\mathbf{p}_{2}\left|H_{\text {int }}^{(s)}(\tau)\right| \mathbf{p}_{1}>=\varphi\left(\mathbf{p}_{2}\right) \varphi^{*}\left(\mathbf{p}_{1}\right) f(\tau),
$$

where $f(\tau)$ is some function of $\tau$, and the form factor $\varphi(\mathbf{p})$ has the following asymptotic behaviour for $|\mathbf{p}| \rightarrow \infty$ :

$$
\varphi(\mathbf{p}) \sim \frac{c_{1}}{|\mathbf{p}|^{\alpha}}, \quad(|\mathbf{p}| \rightarrow \infty) .
$$

In this case, the problem can be easily solved by using equation (44). Representing $<\mathbf{p}_{2}|T(z)| \mathbf{p}_{1}>$ in the form

$$
<\mathbf{p}_{2}|T(z)| \mathbf{p}_{1}>=\varphi\left(\mathbf{p}_{2}\right) \varphi^{*}\left(\mathbf{p}_{1}\right) t(z),
$$

from (44) and (45), we get the equation

$$
\frac{d t(z)}{d z}=-t^{2}(z) \int d^{3} k \frac{|\varphi(\mathbf{k})|^{2}}{\left(z-E_{k}\right)^{2}}
$$

with the asymptotic condition

$$
t(z) \underset{|z| \rightarrow-\infty}{\rightarrow} f_{1}(z)+o\left(|z|^{-\beta}\right),
$$

where

$$
f_{1}(z)=i \int_{0}^{\infty} d \tau \exp (i z \tau) f(\tau),
$$

and $E_{k}=\frac{k^{2}}{2 \mu}$. The solution of equation (57) with the initial condition $t(a)=g_{a}$, where $a \in(-\infty, 0)$, is

$$
t(z)=g_{a}\left(1+(z-a) g_{a} \int d^{3} k \frac{|\varphi(\mathbf{k})|^{2}}{\left(z-E_{k}\right)\left(a-E_{k}\right)}\right)^{-1} .
$$

In the case $\alpha>1 / 2$, the function $t(z)$ tends to a constant as $z \rightarrow-\infty$

$$
t(z) \underset{z \rightarrow-\infty}{\rightarrow} \lambda,
$$

where

$$
\lambda=g_{a}\left(1+g_{a} \int d^{3} k \frac{|\varphi(\mathbf{k})|^{2}}{a-E_{k}}\right)^{-1} .
$$

Thus in this case the function $f_{1}(z)$ must tend to $\lambda$ as $z \rightarrow-\infty$. From this and (59) it follows that the only possible form of the function $f(\tau)$ is

$$
f(\tau)=-2 i \lambda \delta(\tau)+f^{\prime}(\tau),
$$

where the function $f^{\prime}(\tau)$ has no such a singularity at the point $\tau=0$ as the 
the form (48) and hence the dynamics generated by this operator is equivalent to the dynamics governed by the Schrödinger equation with the separable potential

$$
<\mathbf{p}_{2}\left|H_{I}\right| \mathbf{p}_{1}>=\lambda \varphi\left(\mathbf{p}_{2}\right) \varphi^{*}\left(\mathbf{p}_{1}\right)
$$

In particular, in this case from (60) and (61) we easily get the well-known expression for the $\mathrm{T}$ matrix in the separable-potential model

$$
<\mathbf{p}_{2}|T(z)| \mathbf{p}_{1}>=\lambda \varphi\left(\mathbf{p}_{2}\right) \varphi^{*}\left(\mathbf{p}_{1}\right)\left(1-\lambda \int d^{3} k \frac{|\varphi(\mathbf{k})|^{2}}{z-E_{k}}\right)^{-1}
$$

Ordinary quantum mechanics does not permit the extension of the above model to the case $\alpha \leq 1 / 2$. Indeed, in the case of such a large-momentum behaviour of the form factors $\varphi(\mathbf{p})$, substituting the interaction Hamiltonian given by (64) into (51) leads to the ultraviolet divergences, and the integral in (65) is not convergent. We will now show that our formalism allows one to extend this model to the case $0<\alpha<1 / 2$. Let us determine the class of the functions $f_{1}(z)$ and correspondingly the value of $\beta$ for which equation (57) has a unique solution having the asymptotic behaviour (58). In the case $\alpha<1 / 2$, the function $t(z)$ given by (60) has the following behaviour for $z \rightarrow-\infty$ :

$$
t(z) \underset{z \rightarrow-\infty}{\rightarrow} b_{1}|z|^{\alpha-1 / 2}+b_{2}|z|^{2 \alpha-1}+o\left(|z|^{2 \alpha-1}\right),
$$

where $b_{1}=-1 / 2 \cos (\alpha \pi) \pi^{-2} c_{1}^{-2}(2 \mu)^{\alpha-3 / 2}$ and $b_{2}=b_{1}|a|^{1 / 2-\alpha}-b_{1}^{2} g_{a}^{-1}$. Here and below we restrict ourselves to the case when $\varphi(\mathbf{p})=c_{1}|\mathbf{p}|^{-\alpha}$. The parameter $b_{1}$ does not depend on $g_{a}$. This means that all solutions of equation (57) have the same leading term in (66), and only the second term distinguishes the different solutions of this equation. Thus in order to obtain a unique solution of equation (57) we must specify the first two terms in the asymptotic behaviour of $t(z)$ for $z \rightarrow-\infty$. From this it follows that the functions $f_{1}(z)$ must be of the form

$$
f_{1}(z)=b_{1}|z|^{\alpha-1 / 2}+b_{2}|z|^{2 \alpha-1}
$$

and $\beta=2 \alpha-1$. Correspondingly the functions $f(\tau)$ must be of the form

$$
f(\tau)=a_{1} \tau^{-\alpha-1 / 2}+a_{2} \tau^{-2 \alpha},
$$

with $a_{1}=-i b_{1} \Gamma^{-1}(1-2 \alpha) \exp [i(-\alpha / 2+1 / 4) \pi], \quad$ and $\quad a_{2}=-b_{2} \Gamma^{-1}(1-$ $2 \alpha) \exp (-i \alpha \pi)$, where $\Gamma(z)$ is the gamma-function. This means that in the case $\alpha<1 / 2$, the generalized interaction operator must be of the form

$$
<\mathbf{p}_{2}\left|H_{i n t}^{(s)}(\tau)\right| \mathbf{p}_{1}>=a_{1} \varphi\left(\mathbf{p}_{2}\right) \varphi^{*}\left(\mathbf{p}_{1}\right) \tau^{-\alpha-1 / 2}+a_{2} \varphi\left(\mathbf{p}_{2}\right) \varphi^{*}\left(\mathbf{p}_{1}\right) \tau^{-2 \alpha} .
$$

Using (56) and (60), for $<\mathbf{p}_{2}|T(z)| \mathbf{p}_{1}>$, we get 
with

$$
N(z)=g_{a}\left(1+(z-a) g_{a} \int d^{3} k \frac{|\varphi(\mathbf{k})|^{2}}{\left(z-E_{k}\right)\left(a-E_{k}\right)}\right)^{-1}
$$

where

$$
g_{a}=\frac{b_{1}^{2}}{b_{1}|a|^{1 / 2-\alpha}+a_{2} \Gamma(1-2 \alpha) \exp (-i \alpha \pi)} .
$$

It can be easily checked that $N(z)$ given by (71) does not depend on the choice of the parameter $a$, and we can let $a \rightarrow-\infty$. Taking into account that

$$
g_{a} \underset{a \rightarrow-\infty}{\rightarrow} b_{1}|a|^{\alpha-\frac{1}{2}}+b_{2}|a|^{2 \alpha-1}+o\left(|a|^{2 \alpha-1}\right),
$$

and letting $a \rightarrow-\infty$ in (71), we get

$$
N(z)=\frac{b_{1}^{2}}{b_{1}(-z)^{1 / 2-\alpha}-b_{2}} .
$$

In order that the operator $T(z)$ given by (70) and (72) satisfy equations (33) and (34) the parameter $b_{2}$ must be real. The same formula for $T(z)$ can be obtained by using equation (37). It can be shown that if $H_{\text {int }}\left(t_{2}, t_{1}\right)$ is of the form (69), then equation (37) has a unique solution having the behaviour (43) with $\epsilon=-2 \alpha$. In the case when $\varphi(\mathbf{p})=c_{1}|\mathbf{p}|^{-\alpha}$, this solution written for $\tilde{T}(\tau)$ is

$$
<\mathbf{p}_{2}|\tilde{T}(\tau)| \mathbf{p}_{1}>=\sum_{n=1}^{\infty} a_{n} \tau^{n(1 / 2-\alpha)-1} \varphi\left(\mathbf{p}_{2}\right) \varphi^{*}\left(\mathbf{p}_{1}\right)
$$

where

$$
a_{n}=a_{2}^{n-1} a_{1}^{2-n} \Gamma^{n-1}(1-2 \alpha) \Gamma^{2-n}(1 / 2-\alpha) \Gamma^{-1}(n / 2-n \alpha) .
$$

Substituting this series into (18) we get $\left\langle\mathbf{p}_{2}|T(z)| \mathbf{p}_{1}\right\rangle$ which can be represented in the form (70). By using (10), (26) and (70), we can construct the evolution operator

$$
\begin{gathered}
<\mathbf{p}_{2}\left|U\left(t, t_{0}\right)\right| \mathbf{p}_{1}>=<\mathbf{p}_{2} \mid \mathbf{p}_{1}>-\frac{i}{2 \pi} \int_{-\infty}^{\infty} d x \\
\times \frac{\exp \left[-i\left(z-E_{p_{2}}\right) t\right] \exp \left[i\left(z-E_{p_{1}}\right) t_{0}\right]}{\left(z-E_{p_{2}}\right)\left(z-E_{p_{1}}\right)} N(z) \varphi\left(\mathbf{p}_{2}\right) \varphi^{*}\left(\mathbf{p}_{1}\right),
\end{gathered}
$$

where $z=x+i y$, and $y>0$. Since $<\mathbf{p}_{2}|T(z)| \mathbf{p}_{1}>$ given by (70) satisfies equations (33), (34) and (35), the evolution operator $U\left(t, t_{0}\right)$ defined by (12) is a unitary operator satisfying the composition law (4).

We have stated the correspondence between the form of the generalized interaction operator and the large-momentum behaviour of the form factor $\varphi(\mathbf{p})$. In the case $\alpha>1 / 2$, the operator $H_{\text {int }}^{(s)}(\tau)$ would necessarily have the 
case $0<\alpha<1 / 2$ (the restriction $\alpha>0$ is necessary for the integral in (60) to be convergent), the only possible form of $H_{\text {int }}^{(s)}(\tau)$ is (69), and hence the interaction generating the dynamics of the system is nonlocal in time. In the Appendix D it is shown that in the case there are normolized vectors in the Hilbert space for which $<\psi_{2}|U(t, 0)| \psi_{1}>$ are not continuous at $t=0$. These vectors represent the states with infinite energy which are not physically realisable and hence the condition $(7)$ is not violated. Nevertheless, the evolution operator $V(t)=U_{s}(t, 0)$ is not continuous and hence the group of these operators has no infinitesimal generator in this case. From this it follows that in this case the time evolution of a state vector is not governed by the Schrödinger equation. The cause of this discontinuity is quite obvious. Indeed, from the point of view of the states with infinite energy any time interval $\delta t$ is infinite and hence the corresponding matrix elements of the evolution operator $U(\delta t, 0)$ must be independent of $\delta t$, i.e. must be constant. As it is shown in Appendix $\mathrm{D}$, in the case $\alpha>1 / 2$ such matrix elements of $U(t, 0)$ being independent of $t$ are zero, and the discontinuity problem does not appear. This problem appears in the case $\alpha<1 / 2$, i.e. in the case when the matrix elements $<\mathbf{k}_{2}\left|U\left(t_{2}, t_{1}\right)\right| \mathbf{k}_{1}>$ as functions of $\mathbf{k}_{1}$ and $\mathbf{k}_{2}$ do not meet the requirements of ordinary quantum mechanics. Thus the cause of the above mentioned lack of continuity of the evolution operator is the "bad" large-momentum behaviour of the form factors $\varphi(\mathbf{p})$.

Let us now show that the evolution operator defined by (71) and (73) satisfies the condition (7). Using (71) and (73), for the matrix element of the evolution operator in the Schrödinger picture $V(t)$, we can write

$$
<\psi_{2}|V(t)| \psi_{1}>=<\psi_{2} \mid \psi_{1}>-\frac{i}{2 \pi} \int_{-\infty}^{\infty} d x \exp (-i z t) \frac{b_{1}^{2} J(z)}{b_{1}(-z)^{1 / 2-\alpha}-b_{2}},
$$

with

$$
J(z)=\int d^{3} k_{1} \int d^{3} k_{2} \frac{\left|c_{1}\right|^{2} \psi_{1}\left(\mathbf{k}_{1}\right) \psi_{2}^{*}\left(\mathbf{k}_{2}\right)}{\left|\mathbf{k}_{1}\right|^{\alpha}\left|\mathbf{k}_{2}\right|^{\alpha}\left(z-E_{k_{1}}\right)\left(z-E_{k_{2}}\right)},
$$

where $z=x+i y, y>0$, and $\psi_{i}(\mathbf{k})=<\mathbf{k} \mid \psi_{i}>, i=1,2$. By using the fact that the functions $\psi_{1}(\mathbf{k})$ and $\psi_{2}(\mathbf{k})$ must be square-integrable, it can be shown that, if the vectors $\left|\psi_{1}\right\rangle$ and $\left|\psi_{2}\right\rangle$ do not represent the above states with infinite energy, then the function $J(z)$ given by (75) is estimated as

$$
J(z) \leq C(1+|z|)^{-1 / 2-\alpha-\epsilon},
$$

where $\epsilon>0$. From this and the properties of Fourier integrals it follows that for the physically realisable states the matrix elements $\left\langle\psi_{2}|V(t)| \psi_{1}>\right.$ given by (74) are continuous functions of time for $-\infty<t<\infty$ and hence the 


\section{Summary and Discussions}

We have constructed a formalism based on the above assumptions of the Feynman formulation and canonical quantum theory. By using the assumption (iii) in the spirit of the Feynman formalism, within the formalism the probability amplitude $<\psi_{2}\left|U\left(t, t_{0}\right)\right| \psi_{1}>$ is represented as a sum of contributions from the evolution processes associated with completely specified instants of the beginning and end of the interaction in a quantum system, and $<\psi_{2}\left|\tilde{S}\left(t_{2}, t_{1}\right)\right| \psi_{1}>$ represents the contribution from the process in the case of which the interaction begins at time $t_{1}$ and ends at time $t_{2}$. We have shown that this representation and the requirement of unitarity for the evolution operator leads to equation (37) for the operator $\tilde{S}\left(t_{2}, t_{1}\right)$ and equations (33),(34) and (35) for the operator $T(z)$ defined by (18). Equation (37) allows one to obtain the operator $\tilde{S}\left(t_{2}, t_{1}\right)$ if the generalized interaction operator $\mathcal{H}_{\text {int }}\left(t_{2}, t_{1}\right)$ which determines the behaviour of $\tilde{S}\left(t_{2}, t_{1}\right)$ in the limit $t_{2} \rightarrow t_{1}$ is specified. It has been shown that the evolution operator constructed in this way is a unitary operator with the group property (4). In the case when $H_{i n t}^{(s)}(\tau)$ is of the form (48), the Schrödinger equation has been shown to result from equation (37) which can be regarded as an equation of motion. In this case the fundamental interaction in the system is instantaneous and the dynamics governed by this equation of motion is equivalent to the Hamiltonian dynamics. At the same time, our formalism permits the generalization to the case when the fundamental interaction in a quantum system being described by the operator $H_{\text {int }}^{(s)}(\tau)$ is nonlocal in time. In this case the dynamics is not equivalent to the dynamics governed by the Schrödinger equation. This is not at variance with Stone's theorem, since, as it has been shown on the exactly solvable model, the evolution operator is not strongly continuous in this case. It satisfies only the more general continuity condition (7). We have stated correspondence between the large-momentum behaviour of the matrix elements of the evolution operator and the form of the generalized interaction operator $H_{i n t}^{(s)}(\tau)$ : If this behaviour satisfies the requirements of conventional quantum theory, then $H_{\text {int }}^{(s)}(\tau)$ must necessarily be of the form (48), and if this large-momentum behaviour does not meet the above requirements, then $H_{i n t}^{(s)}(\tau)$ must be of the form corresponding to the case when the interaction generating the dynamics of a quantum system is nonlocal in time.

The above gives reason to hope that the proposed formalism may open new possibilities for solving the problem of the ultraviolet divergences in quantum field theory. In this connection note the following. Since locality is the main cause of the ultraviolet divergences, it seems natural to resolve 
the problem by the introduction of a nonlocal form factor into the interaction Hamiltonian density. For example, in QED a nonlocal form factor could be introduced by specifying the interaction Hamiltonian density in the form

$$
\mathcal{H}_{I}(x)=\int d^{4} x_{1} \int d^{4} x_{2} F\left(x-x_{1}, x-x_{2}\right) j_{\mu}\left(x_{1}\right) A^{\mu}\left(x_{2}\right)
$$

where $F\left(x-x_{1}, x-x_{2}\right)$ is a covariant form factor, $j_{\mu}(x)$ is the current density operator, and $A_{\mu}(x)$ is the electromagnetic field potential. However, it turned out that the nonlocal form factor cannot be introduced in such a way without losing covariance of the theory. Indeed, for the theory to be relativistically invariant, $\mathcal{H}_{1}(x)$ must satisfy the condition

$$
\mathcal{H}_{1}\left(x_{1}\right) \mathcal{H}_{1}\left(x_{2}\right)=\mathcal{H}_{I}\left(x_{2}\right) \mathcal{H}_{I}\left(x_{1}\right), \quad\left(x_{2}-x_{1}\right)^{2}<0
$$

But, as is well known, the introduction of the nonlocal form factor leads to violating this condition. The reason for these difficulties is quite obvious. Due to the delta function $\delta(\tau)$ the generalized interaction operator given by (48) describes an instantaneous interaction. In nonrelativistic quantum mechanics processes of instantaneous interaction may be nonlocal in space. However, in relativistic quantum theory a local in time process must be also local in space. Thus, in the case when the generalized interaction operator $H_{\text {int }}^{(s)}(\tau)$ has the form (48), the theory is local in nature, and the introduction of a nonlocal form factor leads to the intrinsic inconsistency of the theory. On the other hand, our formalism permits a generalization to the class of the operators $H_{i n t}^{(s)}(\tau)$ that are not of the form (48). In this case the duration time $\tau=t_{2}-t_{1}$ of the fundamental interaction is not zero, and the description of the dynamics of the system becomes fundamentally nonlocal. For example, in QED the operator $H_{\text {int }}\left(t_{2}, t_{1}\right)$ may be specified in the form

$$
H_{\text {int }}\left(t_{2}, t_{1}\right)=\int d^{3} x_{1} \int d^{3} x_{2} \mathcal{H}_{\text {int }}\left(x_{2}, t_{2}, x_{1}, t_{1}\right)
$$

with

$$
\mathcal{H}_{\text {int }}\left(x_{2}, x_{1}\right)=\int d^{4} y_{1} \int d^{4} y_{2} F\left(x_{2}-x_{1}, x_{2}-y_{1}, x_{2}-y_{2}\right) j_{\mu}\left(y_{1}\right) A^{\mu}\left(y_{2}\right) .
$$

Here $F\left(x_{2}-x_{1}, x_{2}-y_{1}, x_{2}-y_{2}\right)$ is a relativistically invariant form factor. The matrix elements $<\psi_{2}\left|\mathcal{H}_{\text {int }}\left(x_{2}, x_{1}\right)\right| \psi_{1}>$ may be interpreted as amplitudes describing processes in which interaction begins at a point $x_{1}$ of space-time and ends at a point $x_{2}$, and the presence of the nonlocal form factor in the expression of $\mathcal{H}_{\text {int }}\left(x_{2}, x_{1}\right)$ seems to be natural. One may hope that the form factor $F\left(x_{2}-x_{1}, x_{2}-y_{1}, x_{2}-y_{2}\right)$ can be chosen in such a way that no 
and, at the same time, solution of Eq.(37) leads to the ordinary renormalized expression for the $\mathrm{S}$ matrix.

Let us now show that the proposed formalism may open new possibilities for describing the low-energy nucleon dynamics. For this purpose, come back to the model considered in Sec.VI. In the connection with the fact that this model is an extension of the ordinary separable-potential model to the case when the separable interaction is nonlocal in both space and time, note that separable potentials are widely used in nuclear physics (see, for example, Refs.[25-30]). One may hope that within this extended model a better agreement with experiment can be achieved.

In the case when the operator $H_{\text {int }}\left(t_{2}, t_{1}\right)$ has such a form that the interaction generating the dynamics of a system in nonlocal in time, the effective interaction operator $B(z)$ is energy-dependent. As has been mentioned above, the energy dependence of the effective operators of hadron-hadron interactions is associated with the quark and gluon degrees of freedom which are not included explicitly in the description of low-energy hadron interaction. It should be noted that, in general, the energy-dependent operator of hadron-hadron interaction cannot be also assumed to be an optical potential, since due to the quark confinement a hadron system can be considered as a closed system, and for conservation of probability one need not to include explicitly in the description the channels associated with the quark and gluon degrees of freedom. A remarkable feature of the proposed formalism is that the operator of interaction may be energy-dependent even in the case of closed systems when the time evolution is unitary. At the same time, the quark and gluon degrees of freedom may manifest themselves in the dependence upon the time duration $\tau=t_{2}-t_{1}$ of the interaction. In this case the generalized interaction operator may be interpreted as an operator whose matrix elements $<\psi_{2}\left|H_{\text {int }}\left(t_{2}, t_{1}\right)\right| \psi_{1}>$ describe transitions between hadron states, and $t_{1}$ and $t_{2}$ are the times between which the quark and gluon degrees of freedom come into play. One may hope that it will become possible to construct such operators by using QCD - inspired quark models.

Taking into account the quark and gluon degrees of freedom is especially important for describing the short-range NN interaction. In this connection note that in the most realistic NN potentials theoretical parts are supplemented with phenomenological short-range parts. Our formalism makes it possible to describe these phenomenological parts in terms of the operator $H_{\text {int }}\left(t_{2}, t_{1}\right)$. This may open new possibilities for reducing the still existing discrepancy between theory and experiment. 


\section{Appendix A}

Represent (24) in the form

$$
<n_{2}\left|R\left(t, t_{0}\right)\right| n_{1}>=\lim _{a \rightarrow \infty}<n_{2}\left|R_{a}\left(t, t_{0}\right)\right| n_{1}>,
$$

where

$$
\begin{gathered}
<n_{2}\left|R_{a}\left(t, t_{0}\right)\right| n_{1}>=-\frac{a}{2 \pi} \int_{t_{0}}^{t} d t_{2} \int_{t_{0}}^{t_{2}} d t_{1} \int_{-\infty}^{\infty} d x \frac{<n_{2}|T(z)| n_{1}>}{z+a} \\
\times \exp \left(i E_{n_{2}} t_{2}\right) \exp \left[-i z\left(t_{2}-t_{1}\right)\right] \exp \left(-i E_{n_{1}} t_{1}\right)
\end{gathered}
$$

and $a$ is real. Integrating over $t_{1}$ and $t_{2}$ (here the integrations over $t_{1}, t_{2}$ and $x_{1}$ may be reversed in order), we get

$$
\begin{aligned}
& <n_{2}\left|R_{a}\left(t, t_{0}\right)\right| n_{1}>=\frac{a}{2 \pi} \int_{-\infty}^{\infty} d x \frac{<n_{2}|T(z)| n_{1}>}{\left(z-E_{n_{2}}\right)\left(z-E_{n_{1}}\right)(z+a)} \\
& \times \exp \left[-i\left(z-E_{n_{2}}\right) t\right] \exp \left[i\left(z-E_{n_{1}}\right) t_{0}\right]+<n_{2}\left|B\left(t, t_{0}\right)\right| n_{1}>,
\end{aligned}
$$

where

$$
<n_{2}\left|B\left(t, t_{0}\right)\right| n_{1}>=\int_{-\infty}^{\infty} d x<n_{2}\left|F\left(z, t, t_{0}\right)\right| n_{1}>
$$

with

$$
\begin{gathered}
<n_{2}\left|F\left(z, t, t_{0}\right)\right| n_{1}>=-\frac{a}{2 \pi} \frac{<n_{2}|T(z)| n_{1}>}{(z+a)} \\
\times\left[\frac{\exp \left[i\left(E_{n_{2}}-E_{n_{1}}\right) t_{0}\right]}{\left(z-E_{n_{2}}\right)\left(z-E_{n_{1}}\right)}-\frac{\exp \left[i\left(E_{n_{2}}-E_{n_{1}}\right) t\right]-\exp \left[i\left(E_{n_{2}}-E_{n_{1}}\right) t_{0}\right]}{\left(z-E_{n_{2}}\right)\left(E_{n_{2}}-E_{n_{1}}\right)}\right] .
\end{gathered}
$$

Taking into account the above analytic properties of $<n_{2}|T(z)| n_{1}>$ and the asymptotic condition (25), we get

$$
\int_{-\infty}^{\infty} d x<n_{2}\left|F\left(z, t, t_{0}\right)\right| n_{1}>=\oint_{c_{1}} d z<n_{2}\left|F\left(z, t, t_{0}\right)\right| n_{1}>=0,
$$

where $z=x+i y, y>0$, and the contour of integration $c_{1}$ consists of the axis $y=$ constant and the upper half of the infinite circle. Thus for $<n_{2}\left|R\left(t, t_{0}\right)\right| n_{1}>$ we have

$$
\begin{gathered}
<n_{2}\left|R\left(t, t_{0}\right)\right| n_{1}>= \\
=\lim _{a \rightarrow \infty}\left(\frac{a}{2 \pi} \int_{-\infty}^{\infty} d x<n_{2}|T(z)| n_{1}>\frac{\exp \left[-i\left(z-E_{n_{2}}\right) t\right] \exp \left[i\left(z-E_{n_{1}}\right) t_{0}\right]}{\left(z-E_{n_{2}}\right)\left(z-E_{n_{1}}\right)(z+a)}\right) \\
=\frac{1}{2 \pi} \int_{-\infty}^{\infty} d x \frac{\exp \left[-i\left(z-E_{n_{2}}\right) t\right] \exp \left[i\left(z-E_{n_{1}}\right) t_{0}\right]}{\left(z-E_{n_{\odot}}\right)\left(z-E_{n_{1}}\right)}<n_{2}|T(z)| n_{1}>.
\end{gathered}
$$




\section{Appendix B}

Let us show that the evolution operators given by (10) and (26) satisfies the composition law (4), provided equation (35) is valid. The right-hand side of (4) can be rewritten as follows:

$$
U_{r}\left(t, t^{\prime}, t_{0}\right) \equiv U\left(t, t^{\prime}\right) U\left(t^{\prime}, t_{0}\right)=\mathbf{1}+i R\left(t, t^{\prime}\right)+i R\left(t^{\prime}, t_{0}\right)-R\left(t, t^{\prime}\right) R\left(t^{\prime}, t_{0}\right) .
$$

Let $t \geq t^{\prime} \geq t_{0}$. By using (26), for $<n_{2}\left|U_{r}\left(t, t^{\prime}, t_{0}\right)\right| n_{1}>$, we get

$$
\begin{gathered}
<n_{2}\left|U_{r}\left(t, t^{\prime}, t_{0}\right)\right| n_{1}>=<n_{2}\left|n_{1}>+i<n_{2}\right| R\left(t, t^{\prime}\right) \mid n_{1}>+ \\
+i<n_{2}\left|R\left(t^{\prime}, t_{0}\right)\right| n_{1}>+<n_{2}\left|D\left(t, t^{\prime}, t_{0}\right)\right| n_{1}>
\end{gathered}
$$

where

$$
\begin{gathered}
<n_{2}\left|D\left(t, t^{\prime}, t_{0}\right)\right| n_{1}>=-\frac{1}{(2 \pi)^{2}} \sum_{n} \int d x_{1} \int d x_{2} \exp \left[-i\left(z_{2}-E_{n_{2}}\right) t\right] \times \\
\times \exp \left[i\left(z_{1}-E_{n_{1}}\right) t_{0}\right] \exp \left[i\left(z_{2}-z_{1}\right) t^{\prime}\right] \frac{<n_{2}\left|T\left(z_{2}\right)\right| n><n\left|T\left(z_{1}\right)\right| n_{1}>}{\left(z_{2}-E_{n_{2}}\right)\left(z_{2}-E_{n}\right)\left(z_{1}-E_{n}\right)\left(z_{1}-E_{n_{1}}\right)},
\end{gathered}
$$

with $z_{1}=x_{1}+i y_{1}, z_{2}=x_{2}+i y, y_{2}>y_{1}$. Assuming that $T(z)$ satisfies equation (35), this equation can be written in the form

$$
\begin{gathered}
<n_{2}\left|D\left(t, t^{\prime}, t_{0}\right)\right| n_{1}>=-\frac{1}{(2 \pi)^{2}} \int d x_{1} \int d x_{2} \times \\
\times \frac{\exp \left[-i\left(z_{2}-E_{n_{2}}\right) t\right] \exp \left[i\left(z_{1}-E_{n_{1}}\right) t_{0}\right] \exp \left[i\left(z_{2}-z_{1}\right) t^{\prime}\right]}{\left(z_{2}-E_{n_{2}}\right)\left(z_{1}-E_{n_{1}}\right)} \\
\times\left[\frac{<n_{2}\left|T\left(z_{1}\right)\right| n_{1}>}{z_{2}-z_{1}}-\frac{<n_{2}\left|T\left(z_{2}\right)\right| n_{1}>}{z_{2}-z_{1}}\right]=\frac{i}{2 \pi} \int d x_{1} \times \\
\times \frac{\exp \left[-i\left(z_{1}-E_{n_{2}}\right) t\right] \exp \left[i\left(z_{1}-E_{n_{1}}\right) t_{0}\right]-\exp \left[-i\left(z_{1}-E_{n_{2}}\right) t\right] \exp \left[i\left(z_{1}-E_{n_{1}}\right) t^{\prime}\right]}{\left(z_{1}-E_{n_{2}}\right)\left(z_{1}-E_{n_{1}}\right)} \times \\
\times<n_{2}\left|T\left(z_{1}\right)\right| n_{1}>-\frac{i}{2 \pi} \int d x_{2} \frac{\exp \left[-i\left(z_{2}-E_{n_{2}}\right) t^{\prime}\right] \exp \left[i\left(z_{2}-E_{n_{1}}\right) t_{0}\right]}{\left(z_{2}-E_{n_{2}}\right)\left(z_{2}-E_{n_{1}}\right)}<n_{2}\left|T\left(z_{2}\right)\right| n_{1}>= \\
=i\left(<n_{2}\left|R\left(t, t_{0}\right)\right| n_{1}>-<n_{2}\left|R\left(t, t^{\prime}\right)\right| n_{1}>-<n_{2}\left|R\left(t^{\prime}, t_{0}\right)\right| n_{1}>\right) .
\end{gathered}
$$

From this equation it follows that equation (4) is satisfied for $t \geq t^{\prime} \geq t_{0}$. By using (13), it is easy to show then that the evolution operator satisfies the composition law (4) for any $t, t^{\prime}$ and $t_{0}$. 


\section{Appendix C}

Let us now derive an equation directly for the operator $\tilde{S}\left(t_{2}, t_{1}\right)$ starting from equation (35). From this equation, using the definition of $<n_{2}|T(z)| n_{1}>$ given by (27), one can find

$$
\begin{gathered}
\int_{0}^{\infty} d \tau \exp \left(i z_{1} \tau\right) \tau\left(1-\exp \left[i\left(z_{2}-z_{1}\right) \tau\right]\right)<n_{2}|\tilde{T}(\tau)| n_{1}>=\sum_{n} \int_{0}^{\infty} d \tau \exp \left(i z_{1} \tau\right) \\
\times \int_{0}^{\tau} d \tau_{2} \int_{0}^{\tau-\tau_{2}} d \tau_{1}\left(\tau-\tau_{1}-\tau_{2}\right) \exp \left[-i E_{n}\left(\tau-\tau_{1}-\tau_{2}\right)\right]<n_{2}\left|\tilde{T}\left(\tau_{2}\right)\right| n><n\left|\tilde{T}\left(\tau_{1}\right)\right| n_{1}>.
\end{gathered}
$$

For this relation to be valid for any $z_{1}$ and $z_{2}$ the matrix elements $<n_{2}|\tilde{T}(\tau)| n_{1}>$ must satisfy the following equation:

$$
\begin{gathered}
\left(1-\exp \left[i\left(z_{2}-z_{1}\right) \tau\right]\right) \tau<n_{2}|\tilde{T}(\tau)| n_{1}>=\sum_{n} \int_{0}^{\tau} d \tau_{2} \int_{0}^{\tau-\tau_{2}} d \tau_{1}\left(\tau-\tau_{1}-\tau_{2}\right) \\
\times \exp \left[-i E_{n}\left(\tau-\tau_{1}-\tau_{2}\right)\right]<n_{2}\left|\tilde{T}\left(\tau_{2}\right)\right| n><n\left|\tilde{T}\left(\tau_{1}\right)\right| n_{1}>.
\end{gathered}
$$

From this equation, by using (15), one can get the equation (36) for the operator $\tilde{S}\left(t_{2}, t_{1}\right)$. Equation (36) has be rewritten in the form

$$
\begin{gathered}
\left.\left[\sum_{n=1}^{\infty} \frac{(i a)^{n}}{n !}\left(t_{2}-t_{1}\right)^{n}\right)\right] \tilde{S}\left(t_{2}, t_{1}\right)=\int_{t_{1}}^{t_{2}} d t_{4} \int_{t_{1}}^{t_{4}} d t_{3} \\
\times\left[\sum_{m=0}^{\infty} \frac{(i a)^{m}}{m !}\left(t_{2}-t_{4}\right)^{m}\right]\left[\sum_{k=1}^{\infty} \frac{(i a)^{k}}{k !}\left(t_{4}-t_{3}\right)^{k}\right] \tilde{S}\left(t_{2}, t_{4}\right) \tilde{S}\left(t_{3}, t_{1}\right) .
\end{gathered}
$$

For this relation to be valid for every $a$, each term of the expansion in powers of $a$ on the left-hand side of this equation must be equal to the term of the same order on its right-hand side. In the first order, for example, we have

$$
a\left(t_{2}-t_{1}\right) \tilde{S}\left(t_{2}, t_{1}\right)=\int_{t_{1}}^{t_{2}} d t_{4} \int_{t_{1}}^{t_{4}} d t_{3}\left(t_{4}-t_{3}\right) \tilde{S}\left(t_{2}, t_{4}\right) \tilde{S}\left(t_{3}, t_{1}\right) .
$$

From this it follows that $\tilde{S}\left(t_{2}, t_{1}\right)$ must satisfy the equation (37).

\section{Appendix D}

Let us show that in the case $\alpha<1 / 2$ there are states in the Hilbert space for which $<\psi_{2}|U(t, 0)| \psi_{1}>$ are not continuous. Using (10), (12) and (15), for $<\psi_{2}|R(t, 0)| \psi_{1}>$, we can write

$$
<\psi_{2}|R(t, 0)| \psi_{1}>=i \int_{0}^{t} d t_{2} \int_{0}^{t_{2}} d t_{1} \int d^{3} k_{1} \int d^{3} k_{2} \exp \left[i\left(E_{k_{2}}-E_{k_{1}}\right) t_{2}\right] \times
$$


where $\psi_{i}(\mathbf{k})=<\mathbf{k}\left|\psi_{i}\right\rangle, i=1,2$. Consider the vectors $\left|\psi_{\nu}\right\rangle$ for which

$$
<\mathbf{k} \mid \psi_{\nu}>=\frac{\nu^{\frac{1}{2}} d}{k\left(k-\nu-i \nu \Gamma_{0}\right)},
$$

where $d=\Gamma_{0}^{1 / 2}(2 \pi)^{-1 / 2}\left[\pi / 2+\operatorname{arctg}\left(\frac{1}{\Gamma_{0}}\right)^{-1}\right], k=|\mathbf{k}|$, and $\Gamma_{0}$ is a real constant. It is easy to verify that these vectors are normolized.

Let us now consider the case $\alpha>1 / 2$. Taking into account (56) and (63), for $\left\langle\psi_{\nu}|R(t, 0)| \psi_{\nu}>\right.$ in the limit $\nu \rightarrow \infty$, we have

$$
\begin{gathered}
<\psi_{\nu}|R(t, 0)| \psi_{\nu}>=\nu^{1-2 \alpha} g d^{2}\left|c_{1}\right|^{2} \int_{0}^{\theta} d \theta_{1} \int d^{3} q_{1} \int d^{3} q_{2} \times \\
\times \frac{\exp \left[i\left(E_{q_{2}}-E_{q_{1}}\right) \theta_{1}\right]}{\left(q_{1}-1+i \Gamma_{0}\right)\left(q_{2}-1-i \Gamma_{0}\right) q_{1}^{1+\alpha} q_{2}^{1+\alpha}}+o\left(\nu^{1-2 \alpha}\right),
\end{gathered}
$$

where $q_{i}=k_{i} \nu^{-1}, \theta=t \nu^{2}, \theta_{1}=t_{1} \nu^{2}$. It is easy to see that $\left\langle\psi_{\nu}|R(t, 0)| \psi_{\nu}\right\rangle$ given by this equation tends to zero as $\nu \rightarrow \infty$. Let us consider now the case $\alpha<1 / 2$. According to (69), $<\mathbf{k}_{2}|\tilde{T}(\tau)| \mathbf{k}_{1}>$ has the following behaviour near the point $\tau=0$ :

$$
<\mathbf{k}_{2}|\tilde{T}(\tau)| \mathbf{k}_{1}>=a_{1} \varphi\left(\mathbf{k}_{2}\right) \varphi^{*}\left(\mathbf{k}_{1}\right) \tau^{-\beta}+o\left(\tau^{-\beta}\right),
$$

with $\beta=\alpha+1 / 2$. Taking into account this fact, for $\left\langle\psi_{\nu}|R(t, 0)| \psi_{\nu}>\right.$ in the limit $\nu \rightarrow \infty$, we get

$$
<\psi_{k_{0}}|R(t, 0)| \psi_{k_{0}}>=\nu^{2 \beta-1-2 \alpha} \int_{0}^{\theta} d \theta_{2} \int_{0}^{\theta_{2}} d \theta_{1} F_{2}\left(\theta_{1}, \theta_{2}\right)+o\left(\nu^{\alpha+1 / 2-\beta}\right),
$$

where

$$
\begin{gathered}
F_{2}\left(\theta_{1}, \theta_{2}\right)=a_{1} d^{2}\left|c_{1}\right|^{2} \int d^{3} q_{1} \int d^{3} q_{2} \frac{\exp \left[i\left(E_{q_{2}}-E_{q_{1}}\right) \theta_{2}\right] \exp \left[i E_{q_{1}}\left(\theta_{2}-\theta_{1}\right)\right]}{\left(q_{1}-1+i \Gamma_{0}\right)\left(q_{2}-1-i \Gamma_{0}\right) q_{1}^{1+\alpha} q_{2}^{1+\alpha}\left(\theta_{2}-\theta_{1}\right)^{\beta}}, \\
\mathbf{q}_{i}=\mathbf{k}_{i} \nu^{-1}, \theta=t_{i} \nu^{2} \text {, and } \theta=t \nu^{2} \text {. Since } \beta=\alpha+1 / 2, \text { it follows from this that } \\
\lim _{\nu \rightarrow \infty}<\psi_{\nu}|R(t, 0)| \psi_{\nu}>=C,
\end{gathered}
$$

where $C=\int_{0}^{\infty} d \theta_{2} \int_{0}^{\infty} d \theta_{1} F_{2}\left(\theta_{1}, \theta_{2}\right)$. Thus, in the limit $\nu \rightarrow \infty$ the matrix element $<\psi_{\nu}|R(t, 0)| \psi_{\nu}>$ is independent of $t$. This means that there are normalized vectors in the Hilbert space for which $<\psi_{2}|U(t, 0)| \psi_{1}>$ does not tend to $\left\langle\psi_{2}\right| \psi_{1}>$ as $t \rightarrow 0$. However, these vectors represent the states with infinite momentum and correspondingly with infinite energy which are not physically realisable and hence the condition (7) is not violated. 


\section{References}

[1] N.N. Bogolyubov and D.V. Shirkov, Introduction to the Theory of Quantized Fields, 3rd.ed. (Wiley, New York, 1979).

[2] M.A. Braun, Zh. Eksp. Teor.Fiz.94, 145 (1988) [Sov.Phys. JETP 67, 2039 (1988)].

[3] Yu.S. Kalashnikova, I.M.Narodetsky, and V.P.Yurov, Yad. Fiz., 49, 632 (1989).

[4] A.G. Baryshnikov, L.D.Blokhintsev, I.M.Narodetsky, and D.A.Savin, Yad. Fiz. 48, 1273(1988).

[5] A.N. Safronov, Teor.Mat.Fiz. 89, 420(1991); Yad.Fiz., 57, 208(1994).

[6] Yu.A. Kuperin, K.A. Makarov, and S.P.Merkuriev, Teor.Mat.Fiz., 75, 431(1988); 76, 242(1989).

[7] A. Abdurakhmanov and A.L. Zubarev, Z.Phys. A 322, 523(1985).

[8] M. Orlowski, Helv.Phys.Acta. 56, 1053(1983).

[9] B.O. Kerbikov, Yad.Fiz. 41, 725(1985); Teor. Mat.Fiz. 65, 379(1985).

[10] Ya.A.Simonov, Phys.Lett. B, 107, 1(1981).

[11] C.R. Chen, G.L.Payne, J.L.Friar, and B.F.Gibson, Phys. Rev. 31, 2266(1985).

[12] R.P. Feynman, Rev. Mod. Phys. 20, 367(1948).

[13] R.P. Feynman and A.R. Hibbs, Quantum Mechanics and Path Integrals (McGraw-Hill, New York, 1965).

[14] R.F.Streater and A.S.Wightman, PCT, Spin and Statistics, And All That. (W.A.Benjamin, New York, 1964).

[15] N.N.Bogolyubov, B.V.Medvedev, and M.K.Polivanov, Theory of Dispersion Relations. (Lawrence Rad. Lab., Berkeley, Calif., 1961).

[16] M. Reed and B. Simon, Methods of Modern Mathematical Physics I (Academic Press, New York, 1972).

[17] R.Kh. Gainutdinov, Yad. Fiz. 37, 464 (1983) [Sov.J.Nucl.Phys. 37, 277(1983)]. 
[18] R.Kh. Gainutdinov, Yad. Fiz. 46, 1271(1987) [Sov.J.Nucl.Phys. 46, 743(1987)].

[19] R.Kh. Gainutdinov, J. Phys. A. 22, 269(1989).

[20] R.Kh. Gainutdinov, Yad. Fiz. 53, 1431(1991) [Sov.J.Nucl.Phys. 53, 885(1991)].

[21] R.Kh. Gainutdinov, Zh. Eksp. Teor. Fiz. 108, 1600 (1995) [Sov. Phys. JETP 81, 877(1995)].

[22] R. Kh. Gainutdinov and A.A.Mutygullina, Yad. Fiz. 60, 938 (1997) [Physics of Atomic Nuclei, 60, 841 (1997)].

[23] R.Kosloff, Ann.Rev.Phys.Chem., 45, 145 (1994).

[24] H.J.Lüdde, A.Henne, T.Kirchner and R.M.Dreizler, J.Phys.B: At.Mol.Opt.Phys. 29, 4423 (1996).

[25] Y.Yamaguchi, Phys.Rev. 95, 1635 (1954).

[24] F.Tabakin, Phys.Rev. 174, 1208 (1968).

[27] J. Haidenbauer and W. Plessas, Phys.Rev. C 30, 1822 (1984).

[28] G.Rupp and J.A.Tjon, Phys.Rev.C 37, 1729 (1988).

[29] T. Alm and G.Röpke, Phys.Rev.C 50, 31 (1994).

[30] S.K.Adhikari and L.Tomio, Phys.Rev.C 51, 70 (1995). 